\title{
FIRST UNAMBIGUOUS DETECTION OF THE RETURN OF PULSATIONS IN THE ACCRETING WHITE DWARF SDSS J074531.92+453829.6 AFTER AN OUTBURST
}

\author{
Anjum S. Mukadam ${ }^{1,2}$, D. M. Townsley ${ }^{3}$, P. Szkody ${ }^{1,2}$, B. T. Gänsicke ${ }^{4}$, D. E. WingeT ${ }^{5,6}$, J. J. Hermes ${ }^{5,6}$, \\ Steve B. Howell ${ }^{7}$, J. Teske ${ }^{8}$, Joseph Patterson ${ }^{9}$, Jonathan Kemp $^{10}$, and Eve Armstrong ${ }^{9}$ \\ ${ }^{1}$ Department of Astronomy, University of Washington, Seattle, WA 98195-1580, USA; anjum@ astro.washington.edu \\ 2 Apache Point Observatory, 2001 Apache Point Road, Sunspot, NM 88349-0059, USA \\ ${ }^{3}$ Department of Physics and Astronomy, The University of Alabama, Tuscaloosa, AL 35487, USA \\ ${ }^{4}$ Department of Physics, University of Warwick, Coventry, CV4 7AL, UK \\ ${ }^{5}$ Department of Astronomy, University of Texas at Austin, Austin, TX 78712, USA \\ ${ }^{6}$ McDonald Observatory, Fort Davis, TX 79734, USA \\ ${ }^{7}$ NOAO, 950 North Cherry Avenue, Tucson, AZ 85719, USA \\ ${ }^{8}$ Department of Astronomy, University of Arizona, 933 N. Cherry Av, Tucson, AZ 85721, USA \\ ${ }^{9}$ Department of Astronomy, Columbia University, 550 West 120th Street, New York, NY 10027, USA \\ ${ }^{10}$ Joint Astronomy Centre, 660 N. A'ohoku Place, University Park, Hilo, HI 96720, USA \\ Received 2010 November 24; accepted 2010 December 22; published 2011 January 31
}

\begin{abstract}
The primary white dwarf of the cataclysmic variable SDSS J074531.92+453829.6 was discovered to exhibit nonradial pulsations in 2006 January. This accreting white dwarf underwent its first recorded dwarf nova outburst in 2006 October, during which its brightness increased by more than 5 mag. A Hubble Space Telescope (HST) ultraviolet spectrum, obtained one year after the outburst, revealed a white dwarf temperature of $16,500 \mathrm{~K}$, hotter than all other known accreting white dwarf pulsators. This implies that the accreting primary white dwarf of SDSS J074531.92+453829.6 was heated to temperatures beyond the instability strip during the outburst. Optical observations acquired a year after the outburst did not reveal any evidence of pulsations, suggesting that the white dwarf had not cooled to quiescence by then. We recently acquired optical high-speed time-series photometry on this cataclysmic variable SDSS J074531.92+453829.6 more than three years after its outburst to find that pulsations have now returned to the primary white dwarf. Moreover, the observed pulsation periods agree with pre-outburst periods within the uncertainties of a few seconds. This discovery is significant because it indicates that the outburst did not affect the interior stellar structure, which governs the observed pulsation frequencies. It also suggests that the surface of the white dwarf has now cooled to quiescence. Using this discovery in addition to the prior $H S T$ temperature measurement of 16,500 K, we have been able to constrain the matter accreted during the 2006 outburst. This is the first time an accreting white dwarf was unambiguously observed to resume pulsating after an outburst.
\end{abstract}

Key words: novae, cataclysmic variables - stars: dwarf novae - stars: individual (SDSS J074531.92+453829.6) stars: oscillations - stars: variables: general - white dwarfs

\section{INTRODUCTION}

Global pulsations in stars provide the only systematic way to study their interiors. Montgomery \& Winget (1999) and Montgomery et al. (2003) showed that non-radial g-mode pulsations penetrate up to the inner $99 \%$ of white dwarf models. Photometric variations consistent with such non-radial pulsations were first discovered in the cataclysmic variable GW Librae in 1998 (Warner \& van Zyl 1998; van Zyl et al. 2004). A unique model fit to the observed periods of the variable white dwarf can allow us to measure the stellar mass, core composition, age, rotation rate, magnetic field strength, and distance (see the review papers Winget \& Kepler 2008; Fontaine \& Brassard 2008). The class of accreting white dwarf pulsators now has a dozen members, revealing a new venue of opportunity to learn about the fundamental stellar parameters of these accreting variable white dwarfs using asteroseismic techniques (Townsley et al. 2004).

The spectrum of an accreting pulsator includes prominent broad absorption lines from the white dwarf as well as central narrow emission features from the accretion disk. When the orbital period of a cataclysmic variable is $\sim 80$ minutes, it is near the evolutionary orbital period minimum, where the rate of mass transfer is theoretically expected to be the smallest $\sim 10^{-11} M_{\odot} \mathrm{yr}^{-1}$ (Kolb \& Baraffe 1999). Due to the low mass transfer rate, the white dwarf is the source of most of the optical light observed from these systems (Townsley \& Bildsten 2002; Szkody et al. 2010).

ZZ Ceti stars are pure hydrogen atmosphere white dwarf pulsators. The ZZ Ceti instability strip, caused by $\mathrm{H}$ ionization, is located within the temperature range $10,800-12,300 \mathrm{~K}$ for $\log g \approx 8$ (Bergeron et al. 2004; Koester \& Holberg 2001; Mukadam et al. 2004; Gianninas et al. 2005). Accreting white dwarfs show a wide range of $\mathrm{He}$ enrichment from the donor, thus requiring that their pulsational instability region be determined independently from the ZZ Ceti strip. Arras et al. (2006) find a $\mathrm{H} / \mathrm{He} \mathrm{I}$ instability strip for accreting model white dwarfs with a blue edge near $12,000 \mathrm{~K}$ for a $0.6 M_{\odot}$ star, similar to the ZZ Ceti strip. For accreting model white dwarfs with a high He abundance ( $>0.38$ ), Arras et al. (2006) find an additional hotter instability strip at $\approx 15,000 \mathrm{~K}$ due to He II ionization. For a He abundance higher than 0.48 , these theoretical instability strips essentially merge. Szkody et al. (2002, 2007, 2010) have acquired Hubble Space Telescope (HST) UV time-series spectroscopy of nine accreting white dwarf pulsators to establish the instability strip for accretors empirically. Their results reveal a broad instability strip in the temperature range of 
Table 1

Pre-outburst Pulsation Periods Observed in SDSS 0745+4538 Since its Discovery

\begin{tabular}{|c|c|c|c|c|}
\hline \multirow{2}{*}{$\begin{array}{l}\text { Observation } \\
\text { Date (UTC) }\end{array}$} & \multicolumn{4}{|c|}{ Pulsation Period (s); Amplitude (mma) } \\
\hline & Fundamental $\mathbf{P}$ & First Harmonic $\mathrm{P} / 2$ & Second Harmonic $\mathrm{P} / 3$ & Third Harmonic $\mathrm{P} / 4$ \\
\hline 2005 Oct 14 & $1166 \pm 23 ; 70.4 \pm 6.9$ & $597.4 \pm 9.2 ; 46.8 \pm 6.8$ & & \\
\hline 2005 Nov 30 & $1246 \pm 11 ; 46.1 \pm 4.2$ & $613.8 \pm 4.1 ; 28.2 \pm 4.3$ & & \\
\hline $2005 \operatorname{Dec} 30^{\mathrm{a}}$ & $1204.5 \pm 2.5 ; 51.8 \pm 2.7$ & $606.0 \pm 1.1 ; 28.9 \pm 2.7$ & $403.5 \pm 1.0 ; 14.3 \pm 2.7$ & \\
\hline $2006 \operatorname{Jan} 1^{\mathrm{a}}$ & $1192.7 \pm 5.1 ; 53.3 \pm 3.6$ & $606.3 \pm 2.6 ; 26.4 \pm 3.6$ & & \\
\hline $2006 \operatorname{Jan} 2^{\mathrm{a}}$ & $1222 \pm 12 ; 72.2 \pm 6.6$ & & & \\
\hline $2006 \mathrm{Jan} 4^{\mathrm{a}}$ & $\mathbf{1 2 3 2 . 9} \pm \mathbf{3 . 0} ; \mathbf{5 0 . 0} \pm \mathbf{3 . 3}$ & $617.1 \pm 1.6 ; 24.4 \pm 3.3$ & $428.4 \pm 1.4 ; 13.4 \pm 3.3$ & \\
\hline $2006 \operatorname{Jan} 5^{\mathrm{a}}$ & $1228.2 \pm 3.0 ; 48.4 \pm 2.9$ & $613.5 \pm 1.3 ; 28.4 \pm 2.9$ & $410.9 \pm 1.3 ; 12.4 \pm 2.9$ & \\
\hline $2006 \operatorname{Jan} 6^{\mathrm{a}}$ & $1201.7 \pm 1.2 ; 55.3 \pm 2.2$ & $599.23 \pm 0.66 ; 25.8 \pm 2.2$ & $399.14 \pm 0.45 ; 16.9 \pm 2.2$ & $299.93 \pm 0.37 ; 11.5 \pm 2.2$ \\
\hline $2006 \operatorname{Jan} 7^{\mathrm{a}}$ & $1201.1 \pm 1.1 ; 53.6 \pm 2.2$ & $598.40 \pm 0.71 ; 21.5 \pm 2.2$ & $399.87 \pm 0.58 ; 11.9 \pm 2.2$ & \\
\hline $2006 \operatorname{Jan} 8^{\mathrm{a}}$ & $1193.1 \pm 1.6 ; 58.9 \pm 2.6$ & $595.7 \pm 1.1 ; 21.6 \pm 2.6$ & $398.33 \pm 0.74 ; 14.1 \pm 2.6$ & \\
\hline $2006 \operatorname{Jan} 9^{\mathrm{a}}$ & $1230.8 \pm 2.0 ; 40.9 \pm 2.8$ & $617.2 \pm 1.0 ; 19.7 \pm 2.8$ & $404.05 \pm 0.78 ; 11.4 \pm 2.8$ & \\
\hline 2006 Jan 20 & $1216.9 \pm 2.7 ; 60.8 \pm 2.7$ & $607.3 \pm 1.7 ; 24.0 \pm 2.7$ & $405.4 \pm 1.0 ; 17.1 \pm 2.7$ & \\
\hline 2006 Jan 21 & $1218.4 \pm 3.4 ; 61.1 \pm 3.0$ & $609.7 \pm 1.8 ; 28.7 \pm 3.0$ & $408.4 \pm 1.2 ; 18.9 \pm 3.0$ & \\
\hline 2006 Jan 22 & $1290 \pm 40 ; 49.4 \pm 6.7$ & & & \\
\hline 2006 Jan 23 & $1248.0 \pm 4.6 ; 44.6 \pm 3.2$ & $629.4 \pm 2.5 ; 21.0 \pm 3.2$ & $418.1 \pm 2.1 ; 11.3 \pm 3.2$ & \\
\hline 2006 Jan 30 & $1230.4 \pm 6.4 ; 54.2 \pm 3.2$ & $611.0 \pm 3.1 ; 27.8 \pm 3.1$ & & \\
\hline
\end{tabular}

Note. ${ }^{\text {a }}$ These data are previously unpublished.

$10,500-15,400 \mathrm{~K}$ consistent with the theoretical expectations of Arras et al. (2006).

Accreting pulsators have probably undergone a few billion years of accretion and thousands of thermonuclear runaways. Studying these systems will allow us to address the following questions: to what extent does accretion affect the white dwarf mass, temperature, and composition and how efficiently is angular momentum transferred to the core of the white dwarf? Constraining the population, mass distribution, and evolution of accreting white dwarfs is also important for studying supernovae Type Ia systematics.

\section{PREVIOUS DATA ON SDSS J074531.92+453829.6}

Using spectra from the Sloan Digital Sky Survey (SDSS), Szkody et al. (2006) discovered that SDSS J074531.92+ 453829.6 (hereafter SDSS 0745+4538; $g=19.05$ ) is a cataclysmic variable. Mukadam et al. (2007) found high-amplitude non-sinusoidal mono-periodic variations of $45-70 \mathrm{mma}^{11}$ from follow-up photometry of the system in 2005 and interpreted them as non-radial $g$-mode pulsations. The longer light curves revealed the first and second harmonics of the pulsation mode present in this mono-periodic variable (see Table 1). Harmonics are indicative of nonlinear pulse shape, introduced physically by relatively thick convection zones (Brickhill 1992b; Brassard et al. 1995; Wu 2001; Montgomery 2005).

Although only a single pulsation period was observed on any given observing night, different nights revealed the excitation of different periods within the range of 1166-1290 s (see Table 1). This phenomenon of observing different periods on different nights is known as amplitude modulation (Kleinman et al. 1998) and is explained below. The eigenfrequencies of a pulsating white dwarf, representative of its fundamental parameters, and stellar structure, change only on evolutionary timescales $\dot{v} \sim 10^{-19} \mathrm{~Hz} \mathrm{~s}^{-1}$ (e.g., Mukadam et al. 2003; Kepler et al. 2005). On the other hand, white dwarfs in cataclysmic variables do not cool monotonically, but get reheated due to continuous ongoing accretion as well as outbursts 11 One milli modulation amplitude (mma) equals $0.1 \%$ amplitude in intensity
or 1.085 mmag. and superoutbursts. Re-heating and subsequent cooling causes the accreting white dwarf pulsators to evolve faster than their isolated counterparts on timescales of $\dot{v} \sim 10^{-12} \mathrm{~Hz} \mathrm{~s}^{-1}$ (see Townsley et al. 2004). Variable white dwarfs exhibit only a handful of all possible eigenfrequencies at any given time. If new eigenmodes get excited in the star and previously excited eigenmodes are entirely suppressed, then the observed pulsation spectrum will show different frequencies (Kleinman et al. 1998). It is not that the pulsation frequencies change, but that the amplitude of excitation can vary from zero to a finite value on short timescales of order a day; hence this phenomenon is called amplitude modulation.

Table 1 shows the pulsation spectra of all the data on SDSS $0745+4538$, acquired prior to its outburst. These data reveal several characteristics that provide additional proof that SDSS $0745+4538$ exhibits non-radial pulsations. The fundamental pulsation periods shown in boldface allow us to group these data as follows.

Group 1: $1192.7 \pm 5.1$ and $1193.1 \pm 1.6$.

Group 2: $1204.5 \pm 2.5,1201.7 \pm 1.2$, and $1201.1 \pm 1.1$.

Group 3: $1222 \pm 12,1216.9 \pm 2.7$, and $1218.4 \pm 3.4$.

Group 4: $1232.9 \pm 3.0,1228.2 \pm 3.0,1230.8 \pm 2.0$, and $1230.4 \pm 6.4$.

Group 5: $1246 \pm 11$ and $1248.0 \pm 4.6$.

Within each group, the periods are consistent with each other within uncertainties. The weighted group averages are 1193.0, 1202.0, 1218.6, 1229.7, and 1247.4, respectively. The last four period averages may reflect adjacent $k$ modes with the same $\ell$ value, while the $1193 \mathrm{~s}$ mode may involve a distinct $\ell$ value. Similar to the work done by Kleinman et al. (1998) on the cool ZZ Ceti G29-38, we are able to demonstrate that although we observe different periods on different nights in SDSS $0745+4538$ due to a high degree of amplitude modulation, the same periodicities repeat themselves over time, revealing an underlying mode structure.

The long pulsation period, high amplitude, non-sinusoidal pulse shape, and observed amplitude modulation of SDSS 0745+ 4538 are all characteristic features of the cool ZZ Ceti pulsators at $11,000 \mathrm{~K}$. Mukadam et al. (2007) estimated a quiescent effective temperature of $11,000 \pm 2000 \mathrm{~K}$ for the accreting primary 
Table 2

Journal of Observations

\begin{tabular}{llccccc}
\hline \hline $\begin{array}{l}\text { Telescope } \\
\text { Aperture }(\mathrm{m})\end{array}$ & $\begin{array}{c}\text { Start Time } \\
(\mathrm{UTC})\end{array}$ & $\begin{array}{c}\text { Duration } \\
(\mathrm{hr})\end{array}$ & $\begin{array}{c}\text { ExpTime } \\
(\mathrm{s})\end{array}$ & $\begin{array}{c}\text { Dead } \\
\text { Time }(\mathrm{s})\end{array}$ & $\begin{array}{c}\text { Number } \\
\text { of Images }\end{array}$ & Filter \\
\hline FTN 2.0 & 2010 Feb 13 11:06:43 & 2.0 & 60 & 29 & 80 & SDSS g \\
KPNO 2.1 & 2010 Mar 4 02:50:41.14 & 0.5 & 30 & 34 & 20 & BG39 \\
KPNO 2.1 & 2010 Mar 4 03:20:34.46 & 6.0 & 45 & 34 & 160 & BG39 \\
KPNO 2.1 & 2010 Mar 5 02:29:36.82 & 7.4 & 45 & 34 & 206 & BG39 \\
MO 2.1 & 2010 Mar 13 01:55:27 & 5.8 & 15 & 0 & 1396 & BG40 \\
\hline
\end{tabular}

white dwarf in SDSS $0745+4538$ using its optical SDSS spectrum. The high uncertainty of this temperature arises due to the contamination of the optical spectrum by the accretion disk component.

\section{CESSATION OF PULSATIONS AFTER THE 2006 OUTBURST}

The outburst of SDSS $0745+4538$ was detected during the Catalina Real-Time Transient Survey (Drake et al. 2009). The survey records ${ }^{12}$ indicate a brightness of 13.8 mag for the star on 2006 October 27; this constitutes an increase in brightness by more than 5 mag over the quiescent value of $g=19.05$.

Ground-based optical and HST ultraviolet data acquired in 2007 November, a year after the outburst, did not reveal any evidence of pulsations (Szkody et al. 2010). The optical data acquired at the McDonald $2.1 \mathrm{~m}$ telescope for four consecutive nights from 2007 October 30 to 2007 November 2 allowed Szkody et al. (2010) to place the non-variability limit at $8.5 \mathrm{mma}$, significantly smaller than the previously observed pulsation amplitudes of 45-70 mma. The HST time-series spectroscopy acquired on 2007 November 1 could be co-added in wavelength to produce an ultraviolet light curve, which indicated a nonvariability limit of $12.3 \mathrm{mma}$. White dwarf pulsation amplitudes are a function of wavelength (Robinson et al. 1995). We expect the ultraviolet amplitudes to be a few to several times the corresponding optical amplitudes for low azimuthal number $\ell$ modes, as is likely in the case of SDSS $0745+4538$ (see Nitta et al. 2000; Szkody et al. 2007). This makes the HST ultraviolet limit more stringent than the optical non-variability limit, ruling out the presence of any high amplitude pulsations in 2007, which are characteristic for SDSS $0745+4538$.

The co-added $H S T$ spectrum acquired at the time indicated an effective temperature of $16,500 \pm 1000 \mathrm{~K}$ (Mukadam et al. 2009; Szkody et al. 2010), hotter than the hottest known accreting pulsator GW Librae at $15,400 \mathrm{~K}$. This implies that the primary white dwarf in SDSS $0745+4538$ was likely heated to temperatures beyond the instability strip during the outburst and had not cooled sufficiently for the return of pulsations even a year later.

\section{RECENT DATA ON SDSS $0745+4538$}

We obtained optical high-speed time-series photometry on SDSS $0745+4538$ using the CCD Imager (CFIM) on the $2.1 \mathrm{~m}$ telescope at Kitt Peak National Observatory (KPNO), and the prime focus time-series photometer Argos (Nather \& Mukadam 2004) on the $2.1 \mathrm{~m}$ Otto Struve telescope at McDonald Observatory (MO). The journal of observations is shown in Table 2. Data were also acquired on the robotic 2.0 m Faulkes Telescope North (FTN) at Haleakala, Hawaii,

12 http://nesssi.cacr.caltech.edu/catalina/20010317/103171460304100162p. html using the instrument Merope; this telescope belongs to the Las Cumbres Observatory Global Telescope network. Since these data were acquired using the SDSS $g$ filter, we used it to obtain a magnitude measurement of $g=18.9 \pm 0.1$; this magnitude is consistent with the quiescent value of $g=19.05 \pm 0.1$ within the uncertainties. ${ }^{13}$

We determine a blue bandpass BG40 magnitude of $18.90 \pm$ 0.10 for SDSS $0745+4538$ from the McDonald $2.1 \mathrm{~m}$ data obtained on 2010 March 13. For the same telescope and instrumental setup, we had measured a magnitude of $18.96 \pm$ 0.10 for SDSS $0745+4538$ on 2007 October 30 and 31. In conjunction with the Catalina Real-Time Transient Survey records, these consistent magnitudes allow us to rule out any significant changes in the brightness of the system from 2007 November to 2010 December.

We used a standard IRAF (Tody 1993) reduction to extract sky-subtracted light curves from the CCD frames using weighted circular aperture photometry ( $\mathrm{O}$ 'Donoghue et al. 2000). After a preliminary reduction, we brought the data to a fractional amplitude scale $(\Delta I / I)$ and converted the midexposure times of the CCD images to Barycentric Coordinated Time (TCB; Standish 1998). Figure 1 shows the reduced light curves and discrete Fourier transforms (DFTs) of all the recently acquired data on SDSS $0745+4538$, along with the respective $3 \sigma$ white noise limits (Kepler 1993; Mukadam et al. 2010). The best-fit periods and amplitudes are shown below in Table 3 . Each night of observation shows a different pulsation period, but the observed periods are in the same range as the discovery data (Mukadam et al. 2007).

\section{IMPLICATIONS OF THE POST-OUTBURST PULSATION DATA}

The thermal timescale at the base of the convection zone dictates the driving frequency in ZZ Ceti pulsators (Brickhill 1992a; Goldreich \& Wu 1999; Wu 2001; Montgomery 2005), as should also be the case for accreting white dwarf pulsators like SDSS $0745+4538$. The base of the convection zone moves deeper in to the star with cooling and the thermal timescale increases. Hence we expect pulsators closer to the red edge of the instability strip to show longer periods compared to the relatively hotter variables closer to the blue edge. This correlation between the mean pulsation period and spectroscopic temperature is well established for the ZZ Ceti class of pulsators (Clemens 1993; Kanaan et al. 2002; Mukadam et al. 2006). The pulsation periods detected during our recent post-outburst observations agree with the corresponding pre-outburst periods within the uncertainties of a few seconds (see Table 4). This suggests that the thermal

\footnotetext{
13 Since SDSS $0745+4538$ is a large amplitude quiescent pulsator, we have quoted the true uncertainty in its single point SDSS magnitude as $0.1 \mathrm{mag}$ instead of the SDSS uncertainty of $0.01 \mathrm{mag}$. The Calar Alto white light observations acquired on 2006 January 20 during quiescence yield a mean magnitude of $18.88 \pm 0.06$.
} 


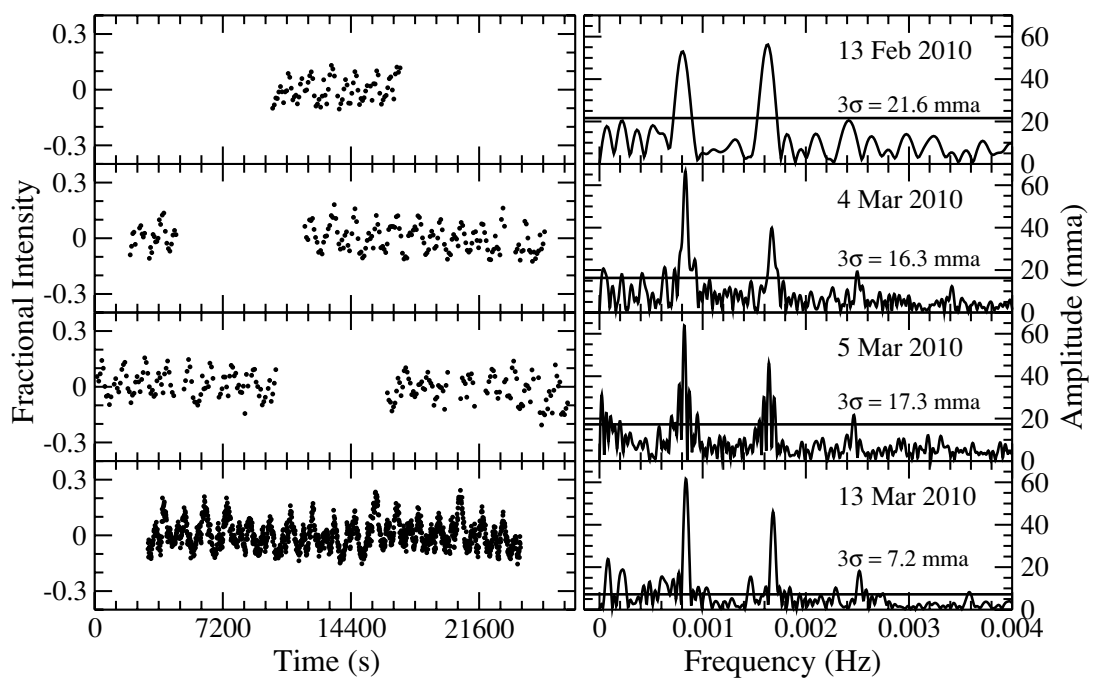

Figure 1. Light curves and corresponding DFTs of the new data on SDSS $0745+4538$.

Table 3

Periodicities Observed in the Light Curves of SDSS 0745+4538

\begin{tabular}{lrrr}
\hline \hline Observation & \multicolumn{3}{c}{ Pulsation Period (s); Amplitude (mma) } \\
\cline { 2 - 4 } Date (UTC) & \multicolumn{1}{c}{ Fundamental P } & \multicolumn{1}{c}{ First Harmonic P/2 } & \multicolumn{1}{c}{ Second Harmonic P/3 } \\
\hline 2010 Feb 13 & $\mathbf{1 2 2 7} \pm \mathbf{1 2} ; \mathbf{5 0 . 0} \pm \mathbf{4 . 8}$ & $616.2 \pm 2.7 ; 53.7 \pm 4.8$ & $411.3 \pm 3.1 ; 20.2 \pm 5.0$ \\
2010 Mar 4 & $\mathbf{1 2 0 2 . 3} \pm \mathbf{2 . 0} ; \mathbf{6 8 . 9} \pm \mathbf{4 . 1}$ & $599.75 \pm 0.81 ; 42.7 \pm 4.0$ & $400.86 \pm 0.99 ; 15.7 \pm 4.0$ \\
2010 Mar 5 & $\mathbf{1 2 1 6 . 5} \pm \mathbf{1 . 7} ; \mathbf{6 4 . 8} \pm \mathbf{4 . 2}$ & $608.43 \pm 0.62 ; 45.4 \pm 4.2$ & $405.89 \pm 0.59 ; 21.2 \pm 4.2$ \\
2010 Mar 13 & $\mathbf{1 1 9 2 . 2} \pm \mathbf{1 . 0} ; \mathbf{6 2 . 3} \pm \mathbf{1 . 7}$ & $594.43 \pm 0.35 ; 46.6 \pm 1.7$ & $396.85 \pm 0.39 ; 18.5 \pm 1.7$ \\
\hline
\end{tabular}

Table 4

Post-outburst Pulsation Periods Match the Corresponding Pre-outburst Values within Uncertainties

\begin{tabular}{lc}
\hline \hline $\begin{array}{l}\text { Post-outburst } \\
\text { Pulsation Period (s) }\end{array}$ & Corresponding Pre-outburst Periods (s) \\
\hline $1227 \pm 12$ & $1232.9 \pm 3.0 ; 1228.2 \pm 3.0 ; 1230.8 \pm 2.0 ; 1230.4 \pm 6.4$ \\
$1202.3 \pm 2.0$ & $1204.5 \pm 2.5 ; 1201.7 \pm 1.2 ; 1201.1 \pm 1.1$ \\
$1216.5 \pm 1.7$ & $1216.9 \pm 2.7 ; 1218.4 \pm 3.4$ \\
$1192.2 \pm 1.0$ & $1192.7 \pm 5.1 ; 1193.1 \pm 1.6$ \\
\hline
\end{tabular}

timescale at the base of the convection zone is about the same in both instances, implying that the stellar surface has now cooled back close to its original quiescent temperature.

Second, the pulsation frequency $\sigma$ of a normal mode is given by the following equation in the absence of any rotational or magnetic field effects:

$$
\sigma_{k, l}=\frac{N \sqrt{\ell(\ell+1)}}{k r},
$$

where $N$ is the Brunt-Vaisälä frequency at the radius $r$, while $k$ and $\ell$ are the radial and azimuthal quantum numbers of the excited mode (see the review paper Winget \& Kepler 2008, and references therein), respectively. Since the post-outburst periods match the pre-outburst values within uncertainties, the above equation also implies that the outburst did not change the Brunt-Vaisälä frequency in the interior. This frequency is given by the difference between the actual and adiabatic density gradients. In other words, fundamental parameters such as the density, pressure, or temperature in the stellar interior were not affected by the outburst of SDSS $0745+4538$.

We have two independent constraints on the degree of cooling that SDSS $0745+4538$ has undergone. The return of pulsations at the same periods as before the outburst implies that the white dwarf has cooled to quiescence in 3.3 years or less. The second constraint on cooling can be inferred from the HST ultraviolet temperature measurement of 16,500 $\pm 1000 \mathrm{~K}$ (Mukadam et al. 2009; Szkody et al. 2010), acquired a year after outburst. The cooling rate of the freshly accreted surface layer is closely related to the total amount of matter accreted during the dwarf nova outburst, $\Delta M_{\mathrm{ob}}$. We will estimate $\Delta M_{\mathrm{ob}}$ using each of these constraints individually.

\subsection{Constraining the Accreted Mass using the HST Temperature}

The asymptotic quiescent effective temperature $T_{\text {eff }, 0}$ indicates the long-term average accretion rate of the primary white dwarf (Townsley \& Bildsten 2003, 2004; Townsley \& Gänsicke 2009). Thus, the degree of cooling observed at a given time interval after outburst, or more precisely the temperature excess over quiescence, allows us to calculate the total amount of matter accreted during the outburst, $\Delta M_{\mathrm{ob}}$. Piro et al. (2005) discuss the cooling of layers immediately below the surface well past an outburst. The excursion of $T_{\text {eff }}$ from the quiescent value at a time $t$ subsequent to the outburst is given by the following equation:

$$
\frac{\delta T_{\text {eff }}}{T_{\text {eff }, 0}}=\left(\frac{t}{t_{\text {late }}}\right)^{-0.81}
$$

where $t_{\text {late }}$ is a characteristic timescale determined by $\Delta M_{\mathrm{ob}}$ and $T_{\text {eff }, 0}$ is the asymptotic quiescent effective temperature. The matter accreted during the dwarf nova outburst is given 
by $\Delta M_{\mathrm{ob}}=4 \pi R^{2} \Delta y$, where $R$ is the white dwarf radius and

$\Delta y=1.8 \times 10^{4} \mathrm{~g} \mathrm{~cm}^{-2} g_{8}^{-2 / 21}\left(\frac{t_{\text {late }}}{1 \text { day }}\right)^{17 / 21}\left(\frac{T_{\text {eff }, 0}}{10,000 \mathrm{~K}}\right)^{60 / 21}$,

with $g_{8}$ being the surface gravity in units of $10^{8} \mathrm{~cm} \mathrm{~s}^{-2}$.

Ideally, multiple measurements are required to determine a reliable value of the parameter $T_{\text {eff }, 0}$. Although this was achieved for the cataclysmic variable WZ Sge by Godon et al. (2004) and Piro et al. (2005), we have to presently contend with an isolated measurement of $T_{\text {eff }}=16,500 \pm 1000 \mathrm{~K}$, obtained a year after outburst. We also have only a pre-outburst optical measurement of the quiescent temperature at $11,000 \pm 2000 \mathrm{~K}$, with twice the uncertainty compared to an HST ultraviolet measurement. Assuming a white dwarf stellar mass of $M=0.6 M_{\odot}$, we derive the following equation:

$$
\begin{aligned}
\Delta M_{\mathrm{ob}}= & 6.9 \times 10^{-9} M_{\odot}\left(\frac{16,500 \mathrm{~K}-T_{\mathrm{eff}, 0}}{16,500 \mathrm{~K}-11,000 \mathrm{~K}}\right) \\
& \times\left(\frac{T_{\mathrm{eff}, 0}}{11,000 \mathrm{~K}}\right)^{39 / 21} .
\end{aligned}
$$

We intend to acquire ultraviolet HST observations of SDSS $0745+4538$ to determine the asymptotic quiescent effective temperature $T_{\text {eff, } 0}$, allowing us to evaluate the above equation and determine a value of the mass accreted during outburst.

\subsection{Constraining the Accreted Mass using the Return of Pulsations}

Heating of the outer envelope enhances the buoyancy in these layers, thereby changing the local Brunt-Vaisälä frequency. This can be mathematically expressed as the Wentzel-Kramers-Brillouin (WKB) integrand $N \propto \sqrt{T}$. Thus, a white dwarf with a heated envelope is expected to show slightly different pulsation periods as the outer accreted layers contribute about $10 \%$ of the above WKB integral (Townsley et al. 2004), which is used to evaluate the eigenmode frequency $\sigma$ (see Equation (1)). Although this contribution depends logarithmically on the accreted mass, we will ignore this dependence for our present preliminary estimate. The temperature excess $\delta T / T$ is approximately constant in the freshly accreted layer (Piro et al. 2005). We therefore estimate that the pulsation frequency $\sigma$ is given by $\sigma / \sigma_{0}=0.9+0.1(1+0.5 \delta T / T)$ during the cooling phase of the accreted layer.

Since the normal mode periods observed 3.3 years after the outburst are consistent with the pre-outburst values within uncertainties, we can place an upper limit on the temperature excess in these outer layers. A $0.3 \%$ uncertainty in period, corresponding to a few seconds for a period around $1200 \mathrm{~s}$, implies an upper limit of $\delta T / T=0.06$. Along with the time since outburst of 3.3 years, this provides another constraint on $t_{\text {late }}$ for the outburst, and therefore on $\Delta M_{\mathrm{ob}}$ :

$$
\Delta M_{\mathrm{ob}} \leqslant 1.9 \times 10^{-9} M_{\odot}\left(\frac{T_{\mathrm{eff}, 0}}{11,000 \mathrm{~K}}\right)^{60 / 21} .
$$

An important caveat to the estimate derived this way is that for some values of the white dwarf spin and radial order of the mode, much of the heated layer may be in a region where oscillation does not propagate (is attenuated). Under such circumstances, heating in that region may have much less influence on the mode than our simplistic and straightforward estimation assumes. Evaluating the actual value of $\sigma / \sigma_{0}$ without this ambiguity is fairly straightforward given the thermal structures discussed by Piro et al. (2005) and Townsley et al. (2004), if enough stellar parameters are known with some confidence. Since we presently lack even an ultraviolet measurement of $T_{\text {eff }, 0}$ for SDSS $0745+4538$, we will leave such a calculation to the future.

\section{DISCUSSION OF THE RESULTS}

Our recent data on SDSS $0745+4538$, acquired three years after its 2006 October outburst, reveal pulsations in the system, making this the first detection of the return of pulsations in an accreting white dwarf after an outburst. We find that the post-outburst pulsation periods agree with the corresponding pre-outburst values from the 2005 discovery data within the uncertainties of a few seconds. Pulsation periods are governed by the inner stellar structure, and the unchanged periods indicate that the outburst did not perturb the white dwarf interior. This argument can also be turned around: since these unaltered frequencies are governed by the stellar interior, we have additional proof that we are dealing with non-radial pulsations as opposed to disk oscillations.

The thermal timescale at the base of the convection zone decides which eigenmodes will be excited in the star. Observing pulsations in the same period range as before the outburst suggests that the surface of the star has now cooled close to quiescence. Once we determine this quiescent temperature using future HST ultraviolet observations, we will be able to evaluate our present constraints on the mass accreted during the 2006 October outburst.

The first accreting white dwarf pulsator to be discovered, GW Librae, underwent an outburst in 2007 April that lasted for 26 days. Copperwheat et al. (2009) suggested the possible return of pulsations in GW Librae 11 months after its outburst when their data revealed periodicities of $1154 \mathrm{~s}$ and $296 \mathrm{~s}$ in the system. However, Bullock et al. (2011) discuss that these periods are actually quasi-periodic oscillations (QPOs) in the accretion disk, while also establishing a continued lack of pulsations in GW Librae three years after its outburst. The outburst of GW Librae was unusual as the $\mathrm{X}$-ray flux at optical maximum was found to be three orders of magnitude above the pre-outburst quiescent level (Byckling et al. 2009), while X-rays are normally suppressed during dwarf nova outbursts. Godon et al. (2006) found WZ Sge $1500 \mathrm{~K}$ above its quiescent temperature three years after its outburst; this cataclysmic variable underwent an outburst for 52 days with an accretion rate of order $10^{-9}$ to $10^{-8} M_{\odot} \mathrm{yr}^{-1}$ and then likely continued accreting at the rate of $10^{-11}$ to $10^{-10} M_{\odot} \mathrm{yr}^{-1}$ during the subsequent cooling phase. Piro et al. (2005) find a $10 \%$ deviation from the quiescent flux even a thousand days after the end of a simulated outburst for a model dwarf nova. However, the accreting white dwarf SDSS $0745+4538$ has returned to quiescence in 3.3 years or less. Unfortunately, the existing data are insufficient to accurately determine the outburst duration or accretion rate, making it difficult to compare directly with observations of other outbursts.

P.S. and A.S.M. thank NSF and NASA for supporting this project through the grants AST-0607840 and HST-GO11163.01-A, respectively. J.P. acknowledges his NSF grant AST-0908363. We thank the Las Cumbres Observatory for time on the $2 \mathrm{~m}$ FTN. 


\section{REFERENCES}

Arras, P., Townsley, D. M., \& Bildsten, L. 2006, ApJ, 643, L119

Bergeron, P., Fontaine, G., Billères, M., Boudreault, S., \& Green, E. M. 2004, ApJ, 600, 404

Brassard, P., Fontaine, G., \& Wesemael, F. 1995, ApJS, 96, 545

Brickhill, A. J. 1992a, MNRAS, 259, 519

Brickhill, A. J. 1992b, MNRAS, 259, 529

Bullock, E., et al. 2011, AJ, in press (arXiv:1101.1238)

Byckling, K., Osborne, J. P., Wheatley, P. J., Wynn, G. A., Beardmore, A., Braito, V., Mukai, K., \& West, R. G. 2009, MNRAS, 399, 1576

Clemens, J. C. 1993, PhD thesis, Univ. of Texas at Austin

Copperwheat, C. M., et al. 2009, MNRAS, 393, 157

Drake, A. J., et al. 2009, ApJ, 696, 870

Fontaine, G., \& Brassard, P. 2008, PASP, 120, 1043

Gianninas, A., Bergeron, P., \& Fontaine, G. 2005, ApJ, 631, 1100

Godon, P., Sion, E. M., Cheng, F., Gänsicke, B. T., Howell, S., Knigge, C., Sparks, W. M., \& Starrfield, S. 2004, ApJ, 602, 336

Godon, P., Sion, E. M., Cheng, F., Long, K. S., Gänsicke, B. T., \& Szkody, P. 2006, ApJ, 642, 1018

Goldreich, P., \& Wu, Y. 1999, ApJ, 511, 904

Kanaan, A., Kepler, S. O., \& Winget, D. E. 2002, A\&A, 389, 896

Kepler, S. O. 1993, Balt. Astron., 2, 515

Kepler, S. O., et al. 2005, ApJ, 634, 1311

Kleinman, S. J., et al. 1998, ApJ, 495, 424

Koester, D., \& Holberg, J. B. 2001, in ASP Conf. Ser. 226, 12th European Workshop on White Dwarfs, ed. J. L. Provencal et al. (San Francisco, CA: ASP), 299

Kolb, U., \& Baraffe, I. 1999, MNRAS, 309, 1034

Montgomery, M. H. 2005, ApJ, 633, 1142

Montgomery, M. H., Metcalfe, T. S., \& Winget, D. E. 2003, MNRAS, 344, 657

Montgomery, M. H., \& Winget, D. E. 1999, ApJ, 526, 976

Mukadam, A. S., Gänsicke, B. T., Szkody, P., Aungwerojwit, A., Howell, S. B., Fraser, O. J., \& Silvestri, N. M. 2007, ApJ, 667, 433
Mukadam, A. S., Montgomery, M. H., Winget, D. E., Kepler, S. O., \& Clemens, J. C. 2006, ApJ, 640, 956

Mukadam, A. S., Szkody, P., Gänsicke, B. T., \& Nitta, A. 2009, J. Phys. Conf. Ser., 172, 012069

Mukadam, A. S., Winget, D. E., von Hippel, T., Montgomery, M. H., Kepler, S. O., \& Costa, A. F. M. 2004, ApJ, 612, 1052

Mukadam, A. S., et al. 2003, ApJ, 594, 961

Mukadam, A. S., et al. 2010, ApJ, 714, 1702

Nather, R. E., \& Mukadam, A. S. 2004, ApJ, 605, 846

Nitta, A., Kanaan, A., Kepler, S. O., Koester, D., Montgomery, M. H., \& Winget, D. E. 2000, Balt. Astron., 9, 97

O’Donoghue, D., Kanaan, A., Kleinman, S. J., Krzesinski, J., \& Pritchet, C. 2000, Balt. Astron., 9, 375

Piro, A. L., Arras, P., \& Bildsten, L. 2005, ApJ, 628, 401

Robinson, E. L., et al. 1995, ApJ, 438, 908

Standish, E. M. 1998, A\&A, 336, 381

Szkody, P., Gänsicke, B. T., Howell, S. B., \& Sion, E. M. 2002, ApJ, 575, L79

Szkody, P., et al. 2006, AJ, 131, 973

Szkody, P., et al. 2007, ApJ, 658, 1188

Szkody, P., et al. 2010, ApJ, 710, 64

Tody, D. 1993, in ASP Conf. Ser. 52, Astronomical Data Analysis Software and Systems II, ed. R. J. Hanisch, R. J. V. Brissenden, \& J. Barnes (San Francisco, CA: ASP), 173

Townsley, D. M., Arras, P., \& Bildsten, L. 2004, ApJ, 608, L105

Townsley, D. M., \& Bildsten, L. 2002, ApJ, 565, L35

Townsley, D. M., \& Bildsten, L. 2003, ApJ, 596, L227

Townsley, D. M., \& Bildsten, L. 2004, ApJ, 600, 390

Townsley, D. M., \& Gänsicke, B. T. 2009, ApJ, 693, 1007

van Zyl, L., et al. 2004, MNRAS, 350, 307

Warner, B., \& van Zyl, L. 1998, in IAU Symp. 185, New Eyes to See Inside the Sun and Stars, ed. F.-L. Deubner, J. Christensen-Dalsgaard, \& D. Kurtz (Cambridge: Cambridge Univ. Press), 321

Winget, D. E., \& Kepler, S. O. 2008, ARA\&A, 46, 157

Wu, Y. 2001, MNRAS, 323, 248 